\section{CORRESPONDENCIAS FORMALES ENTRE EL TANGRAM Y LA OBRA PICTÓRICA DEL NEOPLASTICISTA BART VAN DER LECK}

\author{
FORMAL CONNECTIONS BETWEEN THE TANGRAM AND THE PICTORIAL WORK OF THE \\ NEOPLASTICIST BART VAN DER LECK
}

\author{
TANGRAMAREN ETA BART VAN DER LECK NEOPLASTIZISTAREN LAN PIKTORIKOAREN \\ ARTEKO HARREMAN FORMALAK
}

RESUMEN: Si el reto creativo ante un Tangram es reconstruir distintas figuras en base a un número de piezas invariables y geométricas, esta misma actitud será la que deba asumir el espectador ante la obra del neoplasticista holandés Bart van der Leck, al aplicar una metodología reduccionista y planimétrica, especialmente al geometrizar los temas figurativos y simplificarlos a elementos sueltos en brillantes colores sobre fondo blanco. Esta aportación metodológica conllevará influencias en los trabajos de los demás miembros del grupo De Stijl, particularmente en la obra de Piet Mondrian.

PALABRAS CLAVE: van der Leck; Tangram; neoplasticismo; juegos; geometría.
ABSTRACT: If the target before the Tangram is to reconstruct different silhouettes by means of irregular and geometric pieces, the behavior would be similar for the viewer before the work of Dutch neoplasticist Bart van der Leck, applying a reductionist and planimetric methodology, especially when he abstracted the figurative subjects, in a geometric way and then he reduced them to loose items in bright colors on white background. This methodological contribution had influences on other members of De Stijl, particularly in the work of Piet Mondrian.

KEYWORDS: van der Leck; Tangram; neoplasticism; joys; geometry.
LABURPENA: Tangram baten aurreko desafioa irudi desberdinak berreraikitzea bada pieza aldaezin eta geometrikoak erabiliz, jarrera berdina ikusle batentzat izango litzateke, Bart van der Leck neoplastizista nederlandarraren lanak ikustean. Artista honek erabiltzen zuen planimetriko eta murriztzaile metodologia bat, bereziki gai figuratiboetatik erreferente abstraktugeometrikoak ateratzen zituenean. Geroago hondo zuriaren gaineko kolore distiratsuak aplikatu ohi zituen, loturik gabeko elementuetara murriztuz. Ekarpen metodologiko honek De Stijl taldean egindako proposamenei mailegu ugari erantsi zizkien, gehienbat Piet Mondrian-en lan piktorikoetan.

GAKO-HITZAK: van der Leck Tangrama; neoplastizismoa jostailuak; geometria.

\section{SARRIUGARTE GÓMEZ, Iñigo}

Universidad del País Vasco (UPV/EHU)

Facultad de Letras

Paseo de la Universidad, 5 01006 Vitoria (Álava)

inigo.sarriugarte@ehu.eus

ORCID ID: 0000-0003-0206-9864

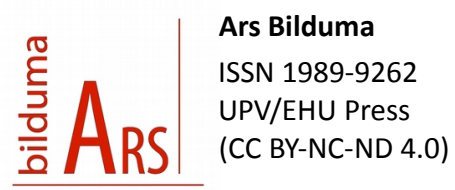

159

https://doi.org/10.1387/ars-bilduma.17633 BIBLID [(2018), 8; 159-175]

Recep.: 08/02/2017 Acept.: 29/05/2017 


\section{BREVE RESEÑA BIOGRÁFICA}

Bart van der Leck nace en Utrecht (1876-1958), posteriormente, a temprana edad, entre 1891 y 1899, comienza a trabajar en talleres de vidrieras, impulsado por la situación económica y los escasos recursos que disponía su familia. Debemos recordar que este campo profesional disponía de una interesante demanda en pedidos y encargos, después de producirse la restauración católica en 1853 en Holanda ${ }^{1}$. La iglesia local en un afán de presentarse ante la sociedad holandesa con gran visualidad y potestad, se embarcó en la construcción de diversos edificios neogóticos, que recaían en arquitectos, mientras que las vidrieras eran derivadas hacia los artistas plásticos, lo que impulsó a van der Leck a estudiar dichas técnicas. De este modo, comenzó a superar las propias barreras que marcaba el marco bidimensional de la pintura y generar de esta manera un diálogo con la arquitectura.

Influenciado e incluso motivado por su anterior y temprano contacto profesional, decide estudiar Artes Aplicadas en Amsterdam de 1901 a 1904, realizando la especialización de arte aplicado sobre cristal y pintura al fresco, mientras acude a las clases nocturnas de la Academia de Bellas Artes de Amsterdam ${ }^{2}$. En un principio, sus trabajos vienen marcados por un carácter simbolista, bajo la influencia de Jan Toorop y Antoon Derkinderen, especialmente en lo que se refiere al estilo de las ilustraciones que elaboró para la edición bibliófila del Cantar de los Cantares. Posteriormente, en 1905 colabora con Piet Klaarhamer en la ilustración de otros libros.

1 BROWN, S. J. \& NOCKLES, P. B. (ed.): The Oxford Movement: Europe and the Wider World 1830-1930. Cambridge, Cambridge University Press, 2012, p. 251

2 CREGO, Ch.: El espejo del orden. El arte y la estética del grupo holandés De Stijl. Madrid, Akal, 1997, p. 43.
Más tarde, se orientará hacia el impresionismo de la Escuela de Amsterdam, bajo la influencia de los paisajes urbanos de George Hendrik Breitner e Isaac Israels ${ }^{3}$. El año 1908 resultará decisivo, ya que tras su contacto con el marchante y crítico de arte Hendricus Petrus Bremmer, empieza a esquematizar y simplificar sus composiciones con figuras de carácter frontal y colores planos. De acuerdo a Hans L.C. Jaffe, van der Leck: "en vez de experimentar con el fauvismo y el cubismo, se unió al grupo de los Monumentalistas Holandeses, cuyo objetivo era realizar en el mural el estilo superpersonal del pasado. En las imágenes de van der Leck, pintadas entre 1908 y 1912, desarrolló un estilo característico de simplificación y estilización, ilustrando libros que le ayudaban a limitar sus términos expresivos de manera estricta." ${ }^{4}$ De hecho, en el trabajo Salida de la fábrica (1910), ya se puede observar como incide en los rasgos fundamentales y generales, eliminando los detalles particulares de los grupos humanos, donde todo se ordena en diferentes líneas y bajo un carácter claramente frontal. Esta simplificación o estilización formal remite a miniaturas medievales y especialmente a relieves egipcios, de hecho, estos ejemplos se encuentran anteriormente en las ilustraciones elaboradas por parte del artista para la edición de 1905 titulada Hooglied van Salomo ${ }^{5}$.

Debemos anotar que van der Leck rara vez solía hacer naturalezas muertas, ya que todo su interés se centraba en la figura humana durante el año 1911. Más adelante, durante 1915, sus composiciones son cada vez más simples, utilizando colores primarios. Por otro lado, su contacto con el

3 WHITE, M.: De Stijl and Dutch Modernism. Manchester, Manchester University Press, 2003, p. 15

4 JAFFE, Hans L.C.: De Stijl. London, Thames and Hudson, 1970, p. 15.

5 BLOTKAMP, C.: Van Gogh to Mondrian: Dutch works on paper. Zwolle, Waanders, Amsterdam, Rijksmuseum (Netherlands); Boston, Museum of Fine Arts, 2000, p. 143. 
crítico de arte y coleccionista H.P. Bremmer y posteriormente con el arquitecto y urbanita Hendrik Petrus Berlage, le proporcionaran los primeros encargos para interiores de la empresa Kröller-Müller ${ }^{6}$ y otro tipo de trabajos como el cartel publicitario de la línea naviera Batavier ${ }^{7}$ (1916). Después de materializar diversos encargos para esta compañía, la señora Kröller-Müller le encomienda la realización de los interiores de su casa de campo, mientras la construcción iba a ser dirigida por H.P. Berlage ${ }^{8}$. A raíz de esta obligada colaboración, y de la consiguiente necesidad de asumir el intercambio de ideas y la conciliación de puntos de vista diversos, la relación entre ambos comenzó a deteriorarse elocuentemente.

Con motivo de su traslado a la localidad de Laren, tiene la oportunidad de conocer a Mondrian, van Doesburg y al carismático matemático y teósofo M.H.J. Schoenmaekers. De acuerdo a Carsten-Peter Warncke, en referencia a Mondrian: "Lejos de la escena artística parisina, en un ambiente provinciano que no favorecía la inspiración, conoció en abril de 1916 a un artista de cuya obra recibiría un importante impulso para superar el Cubismo: Bart van der Leck." ${ }^{\prime 9}$ Gracias a este pintor, Mondrian pudo conocer un sistema de representación abstracto, donde sólo se hacía uso de líneas, colores primarios y tonalidades neutras, a la vez que empleaba superficies bien definidas, siendo su cuadro titulado Composición No. 4 (1916) acogido con gran interés dentro de esta comunidad de artistas; a la vez que atraía de sus cuadros la elaboración rigurosa de formas puramente geométricas.

6 CREGO, op. cit., pp. 47-48.

7 MEGGS, P. B. \& PURVIS, A. W.: Meggs' History of Graphic Design. Hoboken, New Yersey, John Wiley \& Sons, Inc., 2000, láminas 15-43/44

8 WHITE, op. cit., p. 13.

9 WARNCKE, C. P.: El arte de la forma ideal. De Stijl 1917-1931. Köln, Benedikt Taschen, 1993, p. 36.
A partir de 1920, sus trabajos se centran en la aplicación de color para interiores, proyección de vidrieras y diseño textil, mientras que sigue realizando simultáneamente composiciones figurativas.

Durante 1916 y 1917, van der Leck desempeñó en la evolución de De Stijl un papel fundamental, tanto para sí mismo como para los demás. Fue el primero que utilizó los colores primarios en un contexto de planitud espacial; desarrolló la teoría neoplástica y la incorporó sistemáticamente a su propio estilo. Su pertenencia al grupo neoplasticista se produjo más bien en la fase temprana, aunque nunca tuvo un gran interés por pertenecer a éste, debido a las desavenencias mantenidas con otros miembros del grupo, lo que le impulsó a distanciarse de sus principales consideraciones. Entre la fase inicial del grupo neoplasticista y su formación, antes de J.J.P. Oud y Georges Vantongerloo, ya se habían separado del colectivo van der Leck, Jan Wils y Robert van't Hoff ${ }^{10}$. Para el investigador, Carsten-Peter Warncke: "Con todo, la influencia de las soluciones encontradas en común en aquella fase temprana habría de hacerse notar después, de manera que muchas obras de fecha posterior pueden catalogarse entre los ejemplos del diseño típico de Stijl." ${ }^{11}$

10 JOHNSON, D. L. \& LANGMEAD, D.: Makers of 20th-Century Modern Architecture: A BioCritical Sourcebook. London, Fitzroy Dearborn Publishers, 1997, p. 320.

11 WARNCKE, op. cit., p. 111. 


\section{DEFINICIÓN Y CARACTERÍSTICAS DEL TANGRAM}

Durante el siglo XIX, tanto en América como en Europa, comienzan a utilizarse los puzles tridimensionales, procedentes de China, generalmente traídos por los comerciantes de té. Si en un principio se componían de cubos de marfil cortados en piezas irregulares para que se articularan en una caja, otra variante fue el cuadriculado, patentado por Henry Luers a finales del siglo XIX. Este último se componía de 15 piezas diferentes, compuestas por cuadrados que debían encajar para formar un cuadrado único. A partir de aquí, aparecerán nuevas modalidades, al igual que le ocurre al Tangram.

Uno de los juegos más populares de mediados del siglo XIX fueron los paneles de puntos, derivados del décimo don froebeliano ${ }^{12}$, donde se proponía crear dibujos con guisantes, llegando a lograr una fuerte popularidad durante el siglo XX, mediante el fabricante Friedrich Richter $^{13} \mathrm{y}$ su creación Meteor. Estas cajas tenían varios modelos, variando su tamaño, de hecho, con un mayor número de piezas se podían construir más modelos ornamentales. Estas eran pequeñas esferas de color.

12 Debemos recordar que numerosos creadores como Frank Lloyd Wright, Le Corbusier, Paul Klee, Walter Gropius, Josef Albers, Wassily Kandinsky, Piet Mondrian, Theo van Doesburg y George Braque, entre otros habían sido educados en su infancia bajo el sistema de Kindergarten de Froebel, comenzando muchas de sus primeras clases con la realización de dibujos ejecutados mediante triángulos y cubos. Para obtener más información sobre este método, consultar: MORRISON, G. S.: Educación infantil. Madrid, Pearson Educación, 2005, pp. 64-66; BARRIOS, J. L. \& CORDERO, K. (ed.): Grafías en torno a la historia del arte del siglo XX. México, Universidad Iberoamericana, 2006, pp. 50-51.

13 Este fabricante tenía un gran número de cajas de construcción y puzles derivados del tangram.
No se sabe cuándo surge exactamente el Tangram ni quien fue su inventor, aunque el juego es originario de China. De hecho, los primeros libros impresos sobre este divertimiento datan del periodo del emperador Ch'ing Chia Ch'ing (17961820). En chino recibía el nombre de tabla de la sabiduría, tabla de la sagacidad o tabla de los siete elementos ${ }^{14}$. Siguiendo los comentarios de Jaume Llibre, debemos anotar que "en chino, el Tangram se llama tabla de la sabiduría o tabla de los siete elementos. Los dos nombre están bien dados, pues para jugar al Tangram hace falta reflexión y cierta inteligencia." ${ }^{15}$ Debemos recordar la talla que realizó el propio Utamaro en $1880^{16}$, en donde se podían ver a dos cortesanas intentando resolver un problema de Tangram,

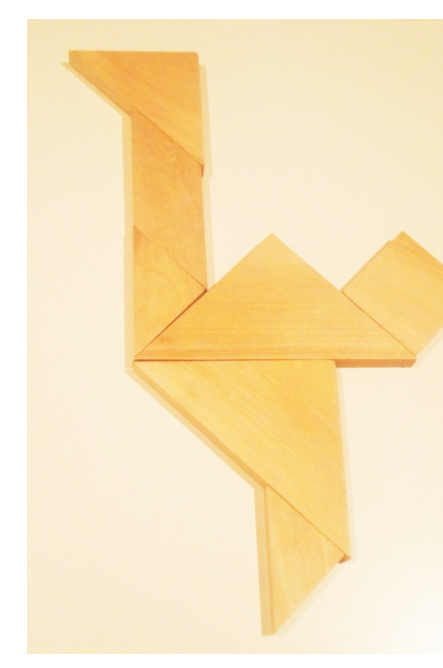

Fig. 1: Elaboración de figuras con el Tangram obteniéndose sus piezas a partir de la partición de un cuadrado, que de hecho al acoplarse de manera adecuada formaba nuevamente esta misma forma. A partir de esta premisa, con siete elementos básicos se establecen todo tipo de figuras generalmente geométricas, situadas en un mismo plano y sin que se superpongan.

Ciertamente, para desarrollar este juego se requiere el uso de la razón lógica y numérica para componer los cientos de formas figurativas que se pueden llegar a elaborar. Se trata únicamente de combinar sus unidades

14 Ch'i ch'ae pan (o juego de los siete elementos). Esta denominación data de la época Chu (740-300 a.d.C), siendo la fecha que se suele tomar como origen del juego.

15 LLIBRE, J.: El Tangram de los ocho elementos. Barcelona, Barral Editores, 1977, p. 12.

16 GRATTAN-GUINNESS, I.: Companion Encyclopaedia of the History and Philosophy of the Mathematical Sciences. London, Taylor \& Francis Books Ltd., 2003, p. 1562. 
básicas cada vez de manera distinta, superando constantemente su forma cuadrada inicial. Se parte de un cuadrado estático para producir todo tipo de movimientos gracias a la combinación de sus elementos (fig. 1). Por este motivo, su uso ha generado un gran interés en el campo de las matemáticas, filosofía, pedagogía, psicología y las artes plásticas.

Como hemos indicado en unas líneas más arriba, si el origen de este entretenimiento se remonta a China, su nombre actual nace en Inglaterra: trangram $^{17}$, significando en el antiguo inglés rompecabezas o juego. A modo de hipótesis, se cree que una mala transcripción de dicha palabra pudo provocar su actual denominación. Otra suposición plantea que la palabra fue originada por un inglés, experto en rompecabezas, quien combina tang, una palabra cantonesa que significa chino, con el sufijo inglés gram (-grama) que significa escrito o gráfico. Incluso, otra versión se basa en que el origen del juego se remontaría al intervalo temporal comprendido entre los años 618 y 907 de nuestra era ${ }^{18}$, cuando dominaba en China la dinastía Tang de donde derivaría su nombre. En cualquier caso, todas las teorías deben ser asumidas como hipótesis, ya que realmente se desconoce con exactitud quien inventó este mecanismo y cuando, aunque como ya hemos indicado anteriormente las primeras publicaciones chinas en las que aparece el juego datan del siglo XVIII.

Este divertimiento fue exportado a Europa en el siglo XIX, donde se difundió con gran rapidez, de hecho, uno de los jugadores más acólitos a

17 JOHNSON, S. \& TODD, H. J. \& CHALMERS, A. \& WALKER, J.: English Dictionary. Boston, Nathan Hale, 1835, p. 940. Como puede apreciarse la conformación del vocablo de este juego mostraba en un principio una ligera variación en relación con su locución actual.

18 ADAMS, C.: Tangram Puzzles: Describing and Comparing Attributes of Plane Geometric Shapes. New York, Rosen Publishing Group, 2004, p. 7. este juego fue Napoleón Bonaparte ${ }^{19}$, desde que sufrió el exilio forzoso en la isla de Santa Elena. Posteriormente, aparecen los primeros libros publicados en relación con el Tangram tanto en América, como en Europa, destaca por ejemplo en 1817-18 un estudio de M. Williams publicado en la revista Leipziger Magazin für Industrie con el título New Mathematical Demostrations of Euclid rendered clear and familiar to the minds of youth, with no other mathematical instruments than the triangular pieces, commonly called the chineses puzzle, donde ya se atisban con claridad las amplias posibilidades educativas, creativas y matemáticas que proyectaba dicho pasatiempo sobre el operador. Según Joost Elffers ${ }^{20}$, las aportaciones posteriores europeas al Tangram fueron el alfabeto y las cifras árabes, desapareciendo los caracteres chinos.

En opinión del investigador Juan Bordes: “El éxito y la difusión de este juego chino fueron tales desde que en 1817 se editaron los primeros manuales de modelos acompañados de las piezas para construirlos que en todo el mundo occidental se habla de la tangranomanía; subyugó a todas las edades y clases sociales, y fue integrado directamente y sin transformaciones como material didáctico en algunos programas de educación infantil. El Tangram fue utilizado de dos maneras: como puzle, con la resolución de figuras propuestas, o como incentivo a la invención, con la creación de nuevas propuestas." ${ }^{21}$ Por otro lado, tal y como afirma

19 Junto a esta figura histórica, también encontramos a otros ilustres tangramistas, como Jonathan Swift, Henry James y Lewis Carroll. También, se especula sobre la supuesta afición a esta actividad por parte de John Quincy Adams y Gustave Doré, siendo más conocido el interés de Edgar Allan Poe por este juego, de hecho, la Biblioteca Pública de Nueva York conserva un juego de tangram de marfil labrado que Poe se hizo importar de China.

20 ELFFERS, J.: El Tangram. Juego de formas chino. Barcelona, Editorial Labor, 1982, p. 30.

21 BORDES, J.: "Jugando a construir la vanguardia: los juguetes comerciales en el siglo XIX derivados de programas educativos", en AA.VV.: Los juguetes de las vanguardias. 
Joost Elffers: "A finales del siglo XIX y comienzos del XX, dos fanáticos de los puzles dedicaron su tiempo y atención al Tangram; fueron éstos Sam Loyd y H. Dudeney. El último colaboró en la revista Strand con una columna de pasatiempos. Sam Loyd es autor de The 8th Book of Tan (El octavo libro de Tan), escrito en 1903. Loyd ideó muchas figuras nuevas sirviéndose para ello, según parece, de las Demostraciones matemáticas de Williams. En El octavo libro de Tan trata de ocultarlo diciendo que el chino Li Hung Chang había demostrado, hace miles de años, el teorema de Pitágoras con ayuda del Tangram. Por cierto que, al igual que Williams, Loyd dividió el cuadrilátero a su manera. Las publicaciones de los últimos años se basan directa o indirectamente en los trabajos de Loyd y Dudeney." ${ }^{22}$

El Tangram consta de siete figuras: un cuadrado, un paralelogramo, cinco triángulos (dos grandes, dos pequeños y uno mediano). Sólo con dichas piezas, no añadiendo ninguna más, se deben construir figuras. Su metodología consiste en formar trazas con todas las piezas sin superponerlas y combinando sus unidades básicas siempre de forma distinta, de hecho, el juego resulta de la descomposición de un polígono regular con una intención específica, donde se puede articular la construcción de cientos de formas figurativas y abstractas. En este sentido, debemos anotar que la simbología del cuadrado ha sido fundamental en todas las culturas, así como en China, de ahí que no sea una casualidad que la división del Tangram se realice en base a un cuadrado. A partir de aquí se han creado otras muchas versiones del juego original ${ }^{23}$, este es el caso del Tangram basado en ocho o cinco piezas, la variante de Fletcher o la modalidad rusa de doce piezas, el cardiotangrama, el ovotangram, el

Málaga, Museo Picasso Málaga, 2010, p. 31.

22 ELFFERS, op. cit., p. 43.

23 Actualmente se pueden llegar a componer con el tangram unas 16.000 figuras distintas. hexatangrama, el armonigrama o tangram pitagórico. Por ejemplo, el armonigrama se emplea para fomentar el apartado algebraico y trabajar con relaciones de orden entre fracciones. Con el cardiotangrama, se trabajan nociones de radio, diámetro, ángulos en el círculo, tangentes, secantes, segmentos circulares, relaciones trigonométricas. Y, por último, por continuar con esta sintomatología de la creatividad matemática, el ovotangrama, que asume forma de huevo, sirve especialmente para construir formas de aves, donde el punto de vista geométrico, se logra asumiendo dos medias elipses en las que el eje menor de la más grande es el eje mayor de la pequeña.

Estos juguetes, que eran muy populares durante el siglo XIX y principios de $X X^{24}$, tuvieron un relevante interés para los futuros arquitectos. En general, no sólo fue un referente en los programas educativos, sino también en la articulación formal y plástica de algunas vanguardias, por ejemplo, debemos destacar los juegos versados en el despiece de sólidos geométricos y los de redes.

El Tangram, tanto en su vertiente de dibujo como puzle, permite mostrar las características cubistas de sus figuras, lo que posibilita un grafismo triangular y cúbico sobre la realidad más orgánica, que evidentemente tendría una relevancia sobre el cubismo y los movimientos abstractos geométricos. La influencia entre ciertos juguetes constructivos y el desarrollo abstracto geométrico perfectamente se podría analizar y tratar, tal y como lo conjetura el experto Juan Bordes: "Aunque sea difícil demostrar una conexión directa de estos conceptos pedagógicos con la

24 En el apartado literario, una de las principales referencias en relación con el tangram, sería la novela Los asesinos chinos (1960) del orientalista holandés Robert van Gulik, donde toda la trama gira en torno a dicho juego. También, merece la pena resaltar el trabajo de Juan Carlos Márquez con el título Tangram. Madrid, Editorial Salto de Página, 2011. 
infancia de todos y cada uno de los grandes arquitectos y artistas fundadores del mundo moderno, fue tan grande su difusión social en toda Europa, América y Rusia que es difícil pensar que los protagonistas de las vanguardias estuvieran aislados de esta atmósfera, ya que su presencia en los ámbitos urbanos y doméstico fue habitual en las grandes ciudades." ${ }^{25}$

\section{CONTIGÜIDADES FORMALES ENTRE EL TANGRAM Y LA OBRA DE VAN DER LECK}

Para el investigador Juan Bordes, muchos de los dispositivos derivados del Tangram tienen cierto parecido a los collages realizados por artistas vanguardistas, siendo "muy semejantes a las obras cubistas de Braque, Picasso o Gris...." ${ }^{26}$, así como a las construcciones abstractas de Schwitters, las propuestas formales y cromáticas de Jean Arp, el mundo de la máquina de Picabia, los gouaches de Matisse y otros tantos ejemplos.

De acuerdo al anterior autor, "el Tangram, citado en su utilización inventiva como origen de algunos juegos de mosaicos, es también en su forma clásica uno de los grupos de puzles de extraordinaria difusión. De esta manera, las cajas con las piezas geométricas producto de una determinada descomposición del cuadrado se presentaban junto a un doble manual donde unas siluetas figurativas masivas se acompañaban con las soluciones que descomponían las mismas figuras en las piezas geométricas que debían construirlas. El carácter cubista de estos modelos transmitió no sólo un grafismo triangular sobre los hábitos visuales, sino también una nueva metodología para la construcción y deconstrucción del dibujo. Y es sólo a

25 BORDES, J.: Historia de los juguetes de construcción. Escuela de los juguetes de construcción. Madrid, Cátedra, 2012, p. 280.

26 BORDES, op. cit., (2010), p. 21. través de este dibujo cómo es posible explicar algunas de las imágenes del libro de recortes que se citaba al comienzo, que presentan una sorprendente coincidencia con los esquemas del cubismo."27

Los juguetes que eran conocidos como mosaicos se subdividían de acuerdo a sus diferencias formales, estando compuestos por mosaicos de piezas cuadradas o cúbicas con división diagonal del color, los mosaicos de teselas planas de geometría variable y los mosaicos derivados del juego chino conocido como Tangram. Este tercer grupo de los mosaicos está compuesto por aquellos que permiten representar figuras en un campo abierto, formando todo tipo de siluetas figurativas sintéticas y cuyo origen es claramente el Tangram ${ }^{28}$. Estos comentarios anteriores se pueden poner en conexión con la metodología de van der Leck: "Esta contribución consistió, por una parte, en su muy personal manera de abstraer los temas figurativos de sus pinturas, de un modo geométrico, y luego reducirlos a elementos sueltos en colores brillantes sobre fondo blanco. Por otra parte, van der Leck estimuló a los otros miembros de De Stijl, particularmente a Mondrian $^{29}$, pintando exclusivamente en suaves planos de colores primarios (considerados objetivos), rojo, azul, y amarillo, en combinación con el uso de blanco, gris y negro." ${ }^{30}$ En esta misma línea, Paul Overy recoge el siguiente comentario de Mondrian, realizado alrededor de 1916:

27 Ibídem, p. 40

28 Ibídem, pp. 30-31.

29 Sobre las conexiones pictóricas y teóricas entre van der Leck y Mondrian, debemos anotar la exposición titulada Piet Mondrian and Bart van der Leck. Inventing a New Art celebrada del 12 de febrero al 21 de mayo de 2017 en el Gemeentemuseum Den Haag. Para obtener más información al respecto, remitirse a la web: https://www.gemeentemuseum.nl/en/exhibitions/piet-mondrian-and-bart-van-derleck. Última consulta: 18-07-2017.

30 PETERSEN, A.: Mondrian, van der Leck, van Doesburg. Obra sobre papel. Valencia, IVAM Centre Julio González, 1991, p. 10. 
“Van der Leck, aunque todavía figurativo, pintaba con planos fijos y colores puros. Mi técnica más o menos cubista - entonces todavía más o menos pictórica - estaba influenciada por su exacta técnica." ${ }^{31}$ Esta actitud anterior se sustenta en que "la operación mental consiste en deconstruir la silueta para hallar la solución, y en el segundo, la imaginación se estimula mediante la construcción." ${ }^{32}$

Van der Leck comenzó a perfeccionar su estilo personal a partir de 1912, asumiendo el fondo de sus imágenes la severidad plana de una pared, lo que permitía eliminar toda sugestión de perspectiva tridimensional. No sólo las figuras aparecían en perfil o delineadas como siluetas, sino que se observaba una evolución hacia formas simplificadas y dominadas por colores primarios y fondos blancos. En este sentido, el año 1916 representa la consolidación de este significativo lenguaje en el uso por parte de van der Leck de los colores y las formas. En la primavera de ese mismo año, completó la pintura Dock Work (fig. 2), en donde se empleaban sólo colores primarios sin mezclar. Debido a que los colores en sí mismos eran bastante convincentes, la línea del contorno en los trabajos iniciales servía para separar los colores pálidos del fondo blanco. Esta propuesta se aborda mediante la esquematización plana, un medio habitual empleado en las artes gráficas, dejando más a un lado la reducción geométrica y lineal. Debemos recordar que Leck había trabajado anteriormente en el diseño de carteles para la línea Batavier de la firma Müller \& $\mathrm{Co}^{33}$, para posteriormente recibir encargos por parte de la dueña de la empresa, la señora Kröller-Müller con el objeto de realizar diseños de vitrales y mosaicos para los edificios de las fábricas.

31 Piet MONDRIAN, citado en OVERY, P.: De Stijl. London, Thames and Hudson, 1991, p. 60

32 BORDES, op. cit., (2010), p. 23.

33 WHITE, op. cit., p. 13.
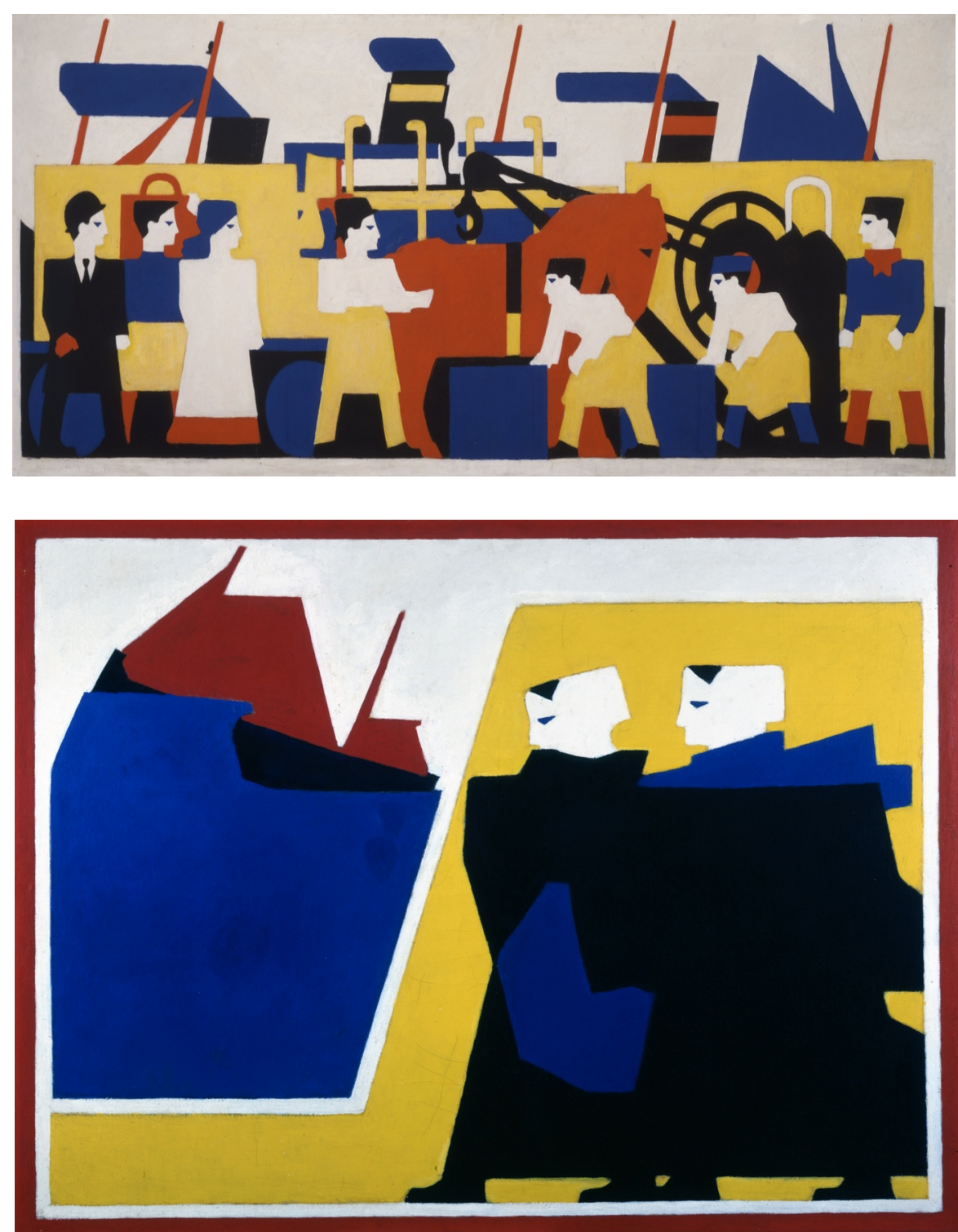

Figs. 2-3: Bart van der Leck: Dock Work; La tempestad, 1916. Kröller-Müller Museum, Otterlo, Holanda 
El cuadro La tempestad (fig. 3) de 1916 representa el clímax de este estilo, donde tomando un fondo a modo de pared blanca, se delimitan unas formas planas firmemente marcadas en colores primarios, que contrastan vivamente unos con otros. Su trabajo viene determinado por una constante simplificación y esquematización de los motivos, convirtiéndose en un revulsivo para las indagaciones pictóricas que estaban llevando a cabo Piet Mondrian, Theo van Doesburg y Vilmos Huszar. También, durante este año, pintó unas cuatro composiciones y un año después subieron a ocho, en cuatro grupos de dos, caso de Coche con dos ruedas (composición 1 y 2), Salida de la fábrica (composición 3 y 4) (fig. 4), Jinetes en burro (composición 5 y 6), Paisajes montañosos (composición 7 y 8). Más adelante, una vez realizado el cuadro $E$ l jinete, a finales de 1918, su pintura retoma definitivamente las pautas de la pintura objetiva.

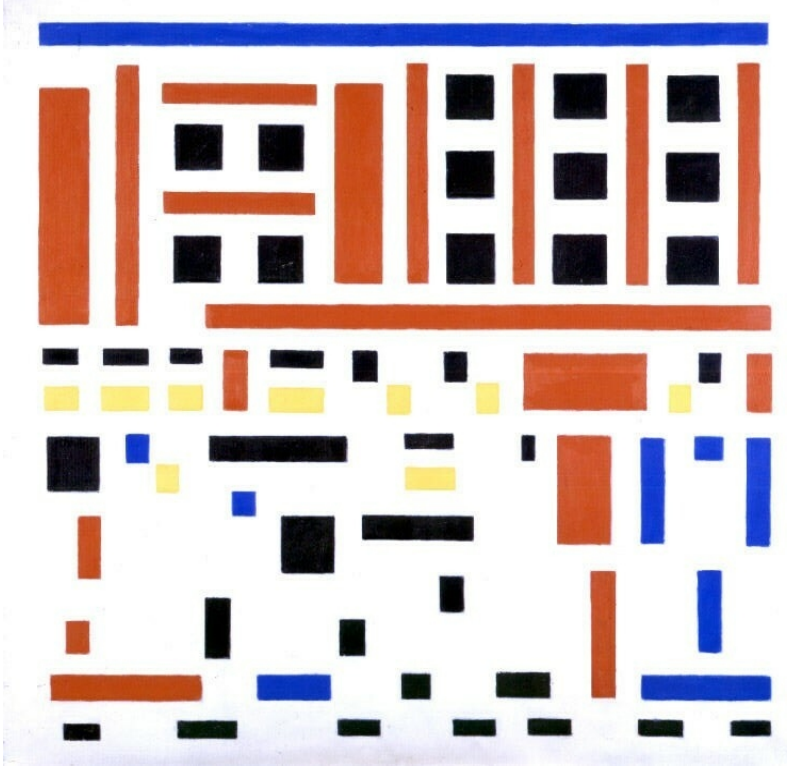

Fig. 4: Bart van der Leck, Salida de la fábrica, Composición no. 4, 1917. Kröller-Müller Museum, Otterlo, Holanda
En 1917, sus propuestas comenzaron a tener una cierta similitud con los trabajos de Mondrian, mediante rectángulos coloreados y líneas fragmentarias distribuidas sobre un fondo blanco, siendo un principio compositivo similar al ritmo empleado por Mondrian en sus trabajos. De acuerdo a van Doesburg: "Si se hace abstracción de las figuras, un cuadro de Ingres o de Poussin es idéntico a un cuadro de Mondrian o de van der Leck. Lo que yo llamo nuevo, o sea acorde con el nivel moral o espiritual de nuestra época, es justamente lo contrario. Lo que nos interesa es la antigravitación, la oblicuidad, un nuevo sentimiento desde el punto de vista del color. La admiración de la disonancia, la aparición de un nuevo espacio, un continuum pictórico en el cual se desarrolla nuestra nueva idea plástica." ${ }^{34}$

El papel de van der Leck en el grupo neoplasticista resultó relevante, ya que fue el primero en emplear colores primarios en base a las coordenadas de una planitud espacial, de hecho, "la geometría era para él la conclusión lógica de su búsqueda de un diseño elemental." ${ }^{35}$ Bajo este proceso de correlación del Tangram con la obra de este artista, debemos anotar las siguientes similitudes: "el Tangram es un juego que tiene un número concreto de elementales formas geométricas de acuerdo con el viejo principio: en la limitación de recursos se ve al maestro. Por lo demás, el jugador tiene entera libertad para operar como quiera. Puede hacerlo solo o con otros. Los diseñadores pueden utilizarlo como auxiliar para hacer esbozos de marcas comerciales y maquetas." ${ }^{36}$ Bajo una aplicación similar, van der Leck trabajaba mediante los mínimos elementos la obtención de un máximo de posibilidades referenciales. En esta misma línea, podemos

34 Theo van DOESBURG, citado en ZEVI, B.: Poética de la arquitectura neoplástica. Buenos Aires, Víctor Lerú, 1960, p. 45.

35 OXENAAR, R. W. D.: “Van der Leck y De Stijl. 1916-1920", en FRIEDMAN, M. (coord.): De Stijl: 1917-1931. Visiones de utopía. Madrid, Alianza Editorial, 1982, p. 78.

36 ELFFERS, op. cit., p. 12 
unir los comentarios de Carsten-Peter Warncke al afirmar lo siguiente: "El mundo pictórico de van der Leck sigue, pues, rindiendo tributo a los objetos, éstos continúan siendo el punto de partida y el punto de referencia de la abstracción. En las series de estudios preliminares que preceden a sus composiciones siempre puede reconocerse claramente el mismo principio, independientemente de los temas que le hayan servido de punta de partida. Mediante una restricción intensificada y sistemática hacia delimitaciones lineales se va creando la composición aparentemente abstracta." ${ }^{37}$ De igual manera que se elabora en la obra de van der Leck, en correlación con este juego, Joost Elffers plantea que "una figura humana corriendo, a la que se le modifique un poco la posición de una pieza, seguirá siendo una figura humana corriendo, pero ya distinta. Desde un punto de vista formal, por este procedimiento se pueden obtener infinitas variantes." ${ }^{38}$

En cualquier caso, una vez desarrolladas estas investigaciones, van der Leck prefirió no ir más allá, anclándose en lo que él mismo consideraba lo vital para su propia evolución artística, claramente marcada por un acercamiento a la realidad circundante. No asumió las proclamas inmovilistas en torno a la negación de la diagonal, tampoco requirió de la profundidad extrema de las especulaciones matemáticas, que otros miembros estaban ensayando, ni siquiera quiso centrarse únicamente en formas rectangulares. Su objetivo ante todo fue representar la realidad visual mediante planos dentro de un movimiento interior del espacio, que perfectamente se podría

37 WARNCKE, op. cit., p. 39.

38 ELFFERS, op. cit., p. 175.

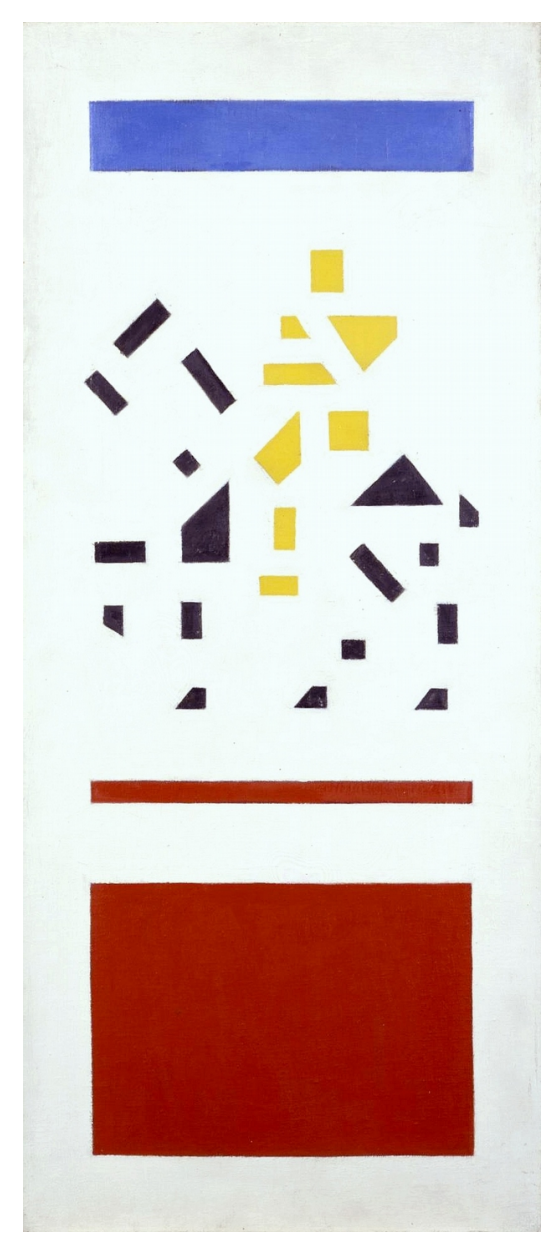

Fig. 5: Bart van der Leck, El Jinete, 1918. Kröller-Müller Museum, Otterlo, Holanda asumir incluso en relación con la cuarta dimensión. Esta interpretación ya ha sido manejada por distintos expertos, caso de Runette Kruger, cuando relaciona la visión utópica y el estudio pictórico de Piet Mondrian con explicaciones de la cuarta dimensión o también llamada filosofía del hiperespacio ${ }^{39}$.

La división del cuadrado, tal y como ocurre en el Tangram, permite construir numerosas composiciones figurativas $y$ abstractas al combinar sus elementos, de hecho, al articular sus unidades básicas cada vez de forma distinta, se procede siempre a generar nuevas posibilidades formativas. Como comenta Jaume Llibre: "los puzles o rompecabezas han tenido siempre un gran número de adeptos. En esencia, un puzle trata siempre de reconstruir una figura que previamente ha sido descompuesta en piezas irregulares, cuyo número es variable... El Tangram chino es un puzle, pero se caracteriza porque la forma y el número de sus piezas son invariables." ${ }^{40}$ En este sentido, van der Leck fue conocido como "un pintor que proporcionaba al espectador una clase de puzzle visual -uno
39 KRUGER, R.: "Art in the Fourth Dimension: Giving Form to Form - The Abstract Paintings of Piet Mondrian", Spaces of Utopia: An Electronic Journal, n. 5, Summer 2007, pp. 23-35. Ubicado en la página web: http://ler.letras.up.pt/uploads/ficheiros/4351.pdf Última consulta: 07-02-2017.

40 LLIBRE, op. cit., p. 9. 
generalmente necesita el título como una pista de lo que está representado. Aunque el visitante medio a menudo obtiene un pequeño vislumbre sobre las motivaciones subyacentes, es uno de los méritos de van der Leck que ha hecho que la abstracción sea inteligible para el público." ${ }^{41}$ Por ejemplo, la imagen que aparece en El Jinete (fig. 5) (1918) de Leck se puede reproducir en el Tangram con formas diversas de jinetes, tal y como lo elabora Roger Lee en su libro ${ }^{42}$. En este sentido, a finales de 1918 recupera las pautas figurativas, sin el menor interés por esconder dichos detalles, de ahí que su anterior trabajo se transforme en la verdadera proclama artística del pintor. A partir de aquí, decide no sólo distanciarse de la práctica del resto de compañeros, sino incluso desde un punto de vista físico, abandona Laren para instalarse en Blaricum, donde había diseñado una casa con su hermano Willem, cuyo interior se organiza como si fuera un volumen blanco con algunos atisbos de colores primarios.

Si la división del Tangram se realiza en base a un cuadrado, dentro del neoplasticismo, el cuadrado se convierte en una de las formas geométricas de principal referencia. En palabras de Joost Elffers, el cuadrado con sus posibilidades estructurales "ha ofrecido a artistas, arquitectos y tendencias estilísticas de todos los tiempos un esquema regular con el que construir una obra de arte" ${ }^{43}$. Por otra parte, debemos anotar la importancia de la simbología ${ }^{44}$ subyacente en torno a esta figura geométrica, que a su vez ha servido para numerosos juegos antiguos, como el ajedrez y las damas. Para el anterior teórico, "el cuadrado es la forma más pura de una superficie,

41 HILHORST, C.: “Bart van der Leck”, en AA.VV.: De Stijl: The Formative Years. 1917-1922. Cambridge, Massachusetts, The Mit Press, 1982, p. 154.

42 LEE, R.: Tangram. Málaga, Sirio, 2009, pp. 37-38.

43 ELFFERS, op. cit., p. 14

44 Resulta de interés la consulta de CONSUEGRA, D.: En busca del cuadrado. Bogotá, Universidad Nacional de Colombia, 1992.

perfecto en sí mismo; todos los demás cuadriláteros son rectángulos que se diferencian en su forma por la altura y la anchura" ${ }^{45}$.

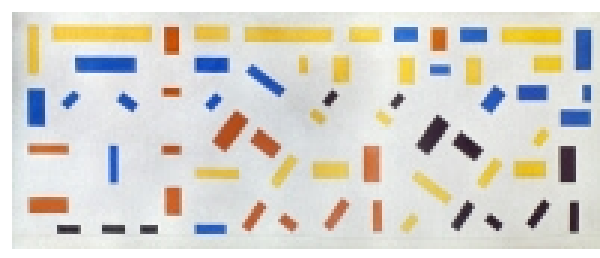

Fig. 6: Bart van der Leck, Jinetes en burro, 1917. The Granger Collection, New York

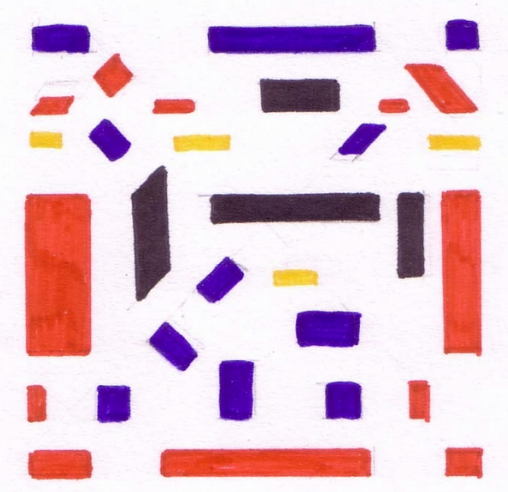

Fig. 7: Bart van der Leck, Composición no 5.1918. Museum de Fundatie, Zwolle and Heino/Wijhe, Holanda
En el número primero de la revista De Stijl se reproducía el cuadro Jinetes en burro (fig. 6) (1917) de Leck, donde se podían ver una serie de planos geométricos, algunos de los cuales estaban en diagonal, a lo que Mondrian en una carta con fecha de 4 de abril de 1918 enviada a van Doesburg comentaba lo siguiente: "También, por lo que respecta a la línea diagonal estoy de acuerdo contigo: creo que se tiene que rechazar cuando aparece de unión con la recta. Van der Leck no sé cómo lo hace, pero sus cosas no dan una impresión individual. Creo que es porque trabaja de una forma totalmente diferente." ${ }^{46}$ Durante 1918, van der Leck utilizaba en sus composiciones planos de rombos,

\footnotetext{
45 ELFFERS, op. cit., p. 14.
}

46 Reproducida en HOEK, E.: “Piet Mondriaan", en BLOTKAMP, C. (ed.): De Beginjaren van De Stijl 1917-1922. Utrecht, Reflex, 1982, p. 69. 
paralelogramos, trapecios, cuadrados, etc. Para este artista, el uso de la diagonal resultaba fundamental, ya que era el mejor medio para la combinación de diferentes fuerzas. Algunas de las obras de Leck de 1918, como Composición no 5, (70 x $70 \mathrm{~cm}$, óleo sobre lienzo) (fig. 7) generaron una fuerte discusión entre los diferentes miembros del grupo debido al empleo de dichas diagonales, que habían sido consideradas como un medio para sugerir la ilusión de espacialidad. El artista dio la misma importancia a las diagonales que a las verticales y horizontales. En este sentido, muchos de los diseños de vitrales de van Doesburg procedían de la manera de trabajar de este último, siguiendo el método de composición geométrico abstracto desarrollado por van der Leck y que, a su vez, se acercaba a la ordenación de dicho juego.

En relación con van Doesburg y aludiendo a un juego infantil, a modo de absurdo irracional e ingenuidad dadaista, debemos anotar la publicación literaria Mecano ${ }^{47}$ (1922-23), cuando este colabora con dicho movimiento, presentando su imagen a través de dos seudónimos: I.K. Bonset y Aldo Camini. Estos seudónimos fueron empleados también por van Doesburg en la revista De Stijl. Con el primer nombre se muestra como poeta dadaísta en 1920; un año más tarde se publican una serie de artículos, que están firmados por Aldo Camini. La revista Mecano, fundada por van Doesburg, se convirtió en el exponente teórico de las ideas dadaístas en Holanda.

47 Para más datos, remitirse a HEDRICK, H. L.: Theo van Doesburg. Propagandist and Practitioner of the Avant-Garde, 1909-1923. Ann Arbor, Michigan, UMI Research Press, 1980, pp. 123-124; YOUNG, A.: Dada and After: Extremist Modernism and English Literature. Manchester, Manchester University Press, 1983, p. 146; DACHY, M.: "Life is an extraordinary invention: Doesburg the dadaist", en FABRE, G. et al. (ed.): Van Doesburg \& the international avant-garde. Constructing a new world. London, Tate Publishing, 2009, pp. 28-35.
Es conocido que el Tangram incentiva estímulos para la creatividad, a la vez que se puede emplear para la enseñanza de las matemáticas y sus conceptos de geometría plana. Evidentemente, incentiva las capacidades psicomotrices e intelectuales, conexionado de un modo lúdico a la manipulación concreta de materiales con la formación de ideas abstractas. Igualmente, permite ejercitarse para mejorar la ubicación espacial, conceptualizar las fracciones y las operaciones entre ellas, operar la aplicación algebraica, deducir fórmulas para área y perímetro de figuras planas. En definitiva, se trata de una introducción al mundo de las matemáticas y la geometría, aspectos que evidentemente tuvieron una notable relevancia en el ámbito neoplasticista y cubista. De hecho, debemos mencionar que los trabajos realizados por parte de van der Leck entre 1917 y 1919 fueron denominados como imágenes matemáticas ${ }^{48}$, afirmando que "la verdad matemática es la expresión de todo lo que existe en el cosmos. En las artes visuales (pintura) una sola imagen matemática o la señal es un símbolo o la imagen de nuestra experiencia espiritual de la realidad cósmica"49. En conexión con esto último y como afirma Robert Vincent, "verdaderamente, el arte no es nada sin el conocimiento y particularmente el conocimiento científico. Un artista es a la vez un artesano, un técnico, un trabajador en el sentido más noble de la palabra." 50

48 EMANUEL, M. et al. (ed.): Contemporary artists. Contemporary arts series. London, Macmillan, 1983, p. 962; DEBBAUT, J. \& VERHULST, M.: Van Abbemuseum: A Companion to Modern and Contemporary Art. Eindhoven, Stedelijk Van Abbemuseum, 2002, p. 49.

49 Bart van der LECK, citado en HILHORST, C.: "Bart van der Leck". Ubicado en la página web: http://www.snap-dragon.com/bart_van_der_leck.htm. Última consulta 06-022017.

50 VINCENT, R.: Geometry of the Golden Section. Marseille, Chalagram Édition, 2007, p. 125. 
Para el teórico Juan Bordes, "algunos de estos Tangram se publicaron con manuales en los que sus figuras eran siluetas de edificios; y uno de los más conocidos fue el firmado por el arquitecto italiano Alessandro Gherardesca (1779-1852) con el título Metamorfosi del giuoco detto l'enimma chinese, editado por Giusepe Landus, en Florencia (1818), con seis páginas de texto y veintitrés láminas que contienen cien modelos en miniatura de villas, logias, iglesias, tumbas, templos y monumentos, puentes y edículos para jardín, que se corresponden con sendas organizaciones de las piezas geométricas del Tangram, demostrando una sorprendente manera de componer la volumetría de los edificios." ${ }^{51}$ Posteriormente, se editaron más manuales por parte del editor londinense Rudolph Ackermann.

En palabras del investigador Juan Bordes: "Es más lógico pensar que fue una educación heterodoxa de la infancia la que acabó por propiciar la atmósfera para que aparecieran los genios artísticos y sociales encargados de elaborar el modelo de la modernidad. De todas formas, esta argumentación quedaría sólo en una opinión fácilmente rebatible si no se aportaran pruebas notariales.... No obstante, el hecho de señalar este origen de la iconografía de las vanguardias no las devalúa en absoluto, aunque si minimiza las interpretaciones que han mitificado $y$ sobrevalorado la novedad como su principal aportación." ${ }^{52}$

Juegos como el Tangram o la linterna mágica tuvieron una notable influencia en algunos de los artistas vanguardistas, especialmente en aquellos que trabajaron e investigaron dentro del cubismo y el neoplasticismo, ya que "el Tangram es un juego planimétrico. Es posible que algunos lo encuentren excesivamente limitado y traten de encontrar ampliaciones tridimensionales, colocando algunas de las piezas en posición

51 BORDES, op. cit., (2012), pp. 314-315.

52 BORDES, op. cit., (2010), p. 51. vertical o diagonal para levantar algo así como castillos de naipes. .... Los elementos básicos del Tangram son planos" ${ }^{\prime 53}$. En esta misma línea, de acuerdo a Paul Overy: "Probablemente ninguno de los trabajos de De Stijl de Van der Leck fueron enteramente abstractos, sino últimamente descompuestos a partir de temas naturalistas, como sus pinturas de 1917. Hasta 1921 alternó pinturas en donde los temas eran fácilmente reconocibles con otros que aparecían casi completamente abstractos. ${ }^{154}$

Si en este mecanismo se produce la composición de una serie de figuras en base a la subdivisión del cuadrado, esta misma aplicación sería administrada por parte de van der Leck, caso de los trabajos titulados $E I$ Bebedor (1919), Mujer y vaca (fig. 8) (1921) y Familia (fig. 9) (1921). El objetivo del Tangram como de las propuestas experimentales de van der Leck era estimular la creatividad, componiendo una realidad de carácter orgánico en geométrica y reducida a su esencia mínima, siguiendo la máxima de geometrizar la naturaleza. Para Carsten-Peter Warncke, "lo que van der Leck trata de comunicar es algo enteramente distinto. Sus cuadros no son, en sentido estricto, abstractos, no-figurativos: más bien se trata de abstracciones que esquematizan o estilizan una figura determinada. Van der Leck nos muestra lo general en lo particular, reduce la técnica de reproducir objetos a sus elementos, y, partiendo de ellos, construye composiciones que a pesar de su carácter abstracto no dejan de referirse al mundo cotidiano de la experiencia." ${ }^{55}$

En base a esta práctica metodológica, se posibilita una serie de características, que no sólo se observan en la obra de van der Leck, sino también en las aportaciones artísticas de otros neoplasticistas: planificar el

53 ELFFERS, op. cit., pp. 11-12.

54 OVERY, op. cit., p. 64.

55 WARNCKE, op. cit., p. 28. 
trazado de la figura sobre la base del análisis de sus propiedades; analizar los efectos que generan en el perímetro o en el área de cuadrados y rectángulos la variación de la medición de sus lados; analizar temas relacionados con la geometría; reproducir figuras y representaciones planas de cuerpos geométricos; combinar figuras para generar otras previas establecidas; calcular perímetro y áreas de figuras compuestas por cuadrados, rectángulos y otros tipos de polígonos; descubrir formulas a partir de los modelos existentes, así como fomentar la creatividad y las capacidades del autoaprendizaje. Este proceso anterior se observa en el comentario de Ad Petersen: "para Mondrian, van der Leck y van Doesburg, el dibujo apenas era un fin en sí mismo, sino habitualmente una etapa intermedia, un esbozo o estudio preliminar para una pintura." 56
No obstante, resulta de interés anotar una de las principales diferencias entre las realizaciones planimétricas del artista holandés y este juego. Tal y como lo relata Joost Elffers, una característica peculiar de las piezas del Tangram sería la siguiente: "una interesante propiedad de los tangramas es su convexidad. Se dice de una figura que es convexa cuando tiene, respecto de quien la mira, la superficie más prominente en el centro y decrece de manera uniforme hacia los bordes o extremos." ${ }^{57}$ En este sentido, la proyección del Tangram no llega a ser absolutamente planimétrica, a pesar de que esta se muestra como una característica general, lo que aparentemente genera una clara similitud con algunas de las composiciones del artista holandés, quedando todas las figuras contenidas en el mismo plano.

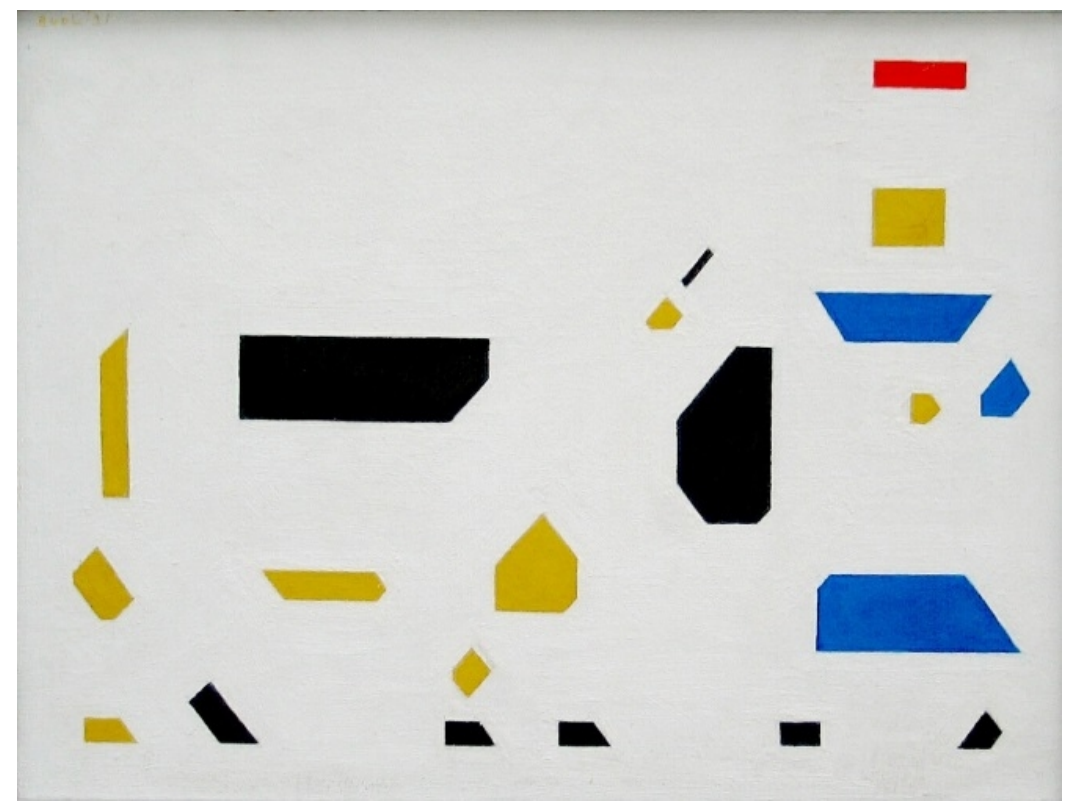

56 PETERSEN, op. cit., p. 9.

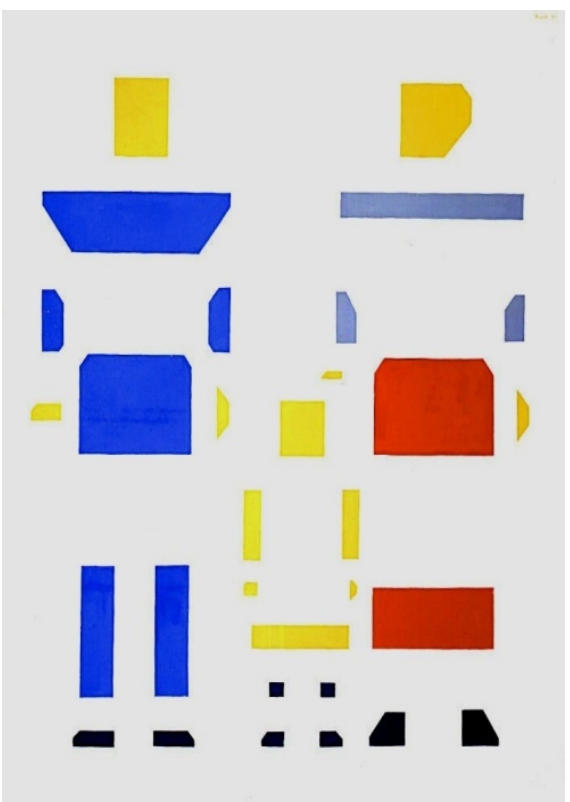

Figs. 8-9: Bart van der Leck: Mujer y vaca; Familia, 1921. Kröller-Müller Museum, Otterlo, Holanda 


\section{CONCLUSIONES}

El tratamiento que obtuvo el artista holandés Bart van der Leck mediante su sistema reductivo y esquematizado de formas planas, expuestas de manera frontal, junto a colores primarios, que tanto llegó a influir en sus contemporáneos neoplasticistas, caso de las propuestas pictóricas de Mondrian y las vidrieras de Theo van Doesburg, asume un acercamiento formal hacia el universo constructivo del Tangram, donde la elaboración de cualquier composición figurativa era articulada mediante la combinación de un grupo de siete figuras, como un cuadrado, un paralelogramo y cinco triángulos de diferentes tamaños. La similitud del proceso de construcción de nuestra naturaleza desde un parámetro geométrico y bajo las coordenadas de una planimetría espacial, llevada a cabo por van der Leck fomenta una semejanza compositiva con las aportaciones que ofrece este juego de origen chino.

Tanto los trabajos pictóricos del artista como los resultados articulados del Tangram ofrecen al espectador una sensación de puzle visual, lo que ha permitido el acercamiento del concepto de la abstracción hacia el espectador. No obstante, las orientaciones formativas se presentan de origen opuesto, ya que mientras el Tangram construye realidades figurativas revelándose desde una base abstracta, van der Leck parte de la observación de una naturaleza orgánica para encaminarse hacia un entramado geométrico que se sitúa en las puertas de la no-objetividad. El recorrido de ambos métodos en sentido inverso infunde resultados similares en determinadas etapas procesuales, lo que permite generar la posibilidad de una práctica comparativa entre ambos temas en base a un método hipotético-deductivo.

Esta similitud formal y compositiva entre las propuestas del artista y las posibilidades ejecutoras del juego nos trasladan a una realidad que interesaba de manera muy visible a los distintos movimientos geométricoabstractos y en especial al neoplasticismo, ya que eliminaba el falso velo que defendía la desconexión entre la abstracción y la figuración, ya que ambos al ser puntos tanto de partida como de inicio, permitían obtener determinados estadios intermedios, donde se conectaban formal y compositivamente a raíz de la aplicación de sus procesos experimentales. 


\section{BIBLIOGRAFÍA}

ADAMS, C.: Tangram Puzzles: Describing and Comparing Attributes of Plane Geometric Shapes, New York, 2004.

BARRIOS, J. L. \& CORDERO, K. (ed.): Grafías en torno a la historia del arte del siglo XX, México, 2006.

BLOTKAMP, C.: Van Gogh to Mondrian: Dutch works on paper, Zwolle, Waanders, 2000.

BORDES, J.: "Jugando a construir la vanguardia: los juguetes comerciales en el siglo XIX derivados de programas educativos", en Los juguetes de las vanguardias, Málaga, 2010.

: Historia de los juguetes de construcción. Escuela de los juguetes de construcción, Madrid, 2012.

BROWN, S. J. \& NOCKLES, P. B. (ed.): The Oxford Movement: Europe and the Wider World 1830-1930, Cambridge, 2012.

CREGO, Ch.: El espejo del orden. El arte y la estética del grupo holandés De Stijl, Madrid, 1997.

DACHY, M.: "Life is an extraordinary invention: Doesburg the dadaist", en Van Doesburg \& the international avant-garde. Constructing a new world, London, 2009.

DEBBAUT, J. \& VERHULST, M.: Van Abbemuseum: A Companion to Modern and Contemporary Art, Eindhoven, 2002.
ELFFERS, J.: El Tangram. Juego de formas chino, Barcelona, 1982.

EMANUEL, M. et al. (ed.): Contemporary artists. Contemporary arts series, London, 1983.

GRATTAN-GUINNESS, I.: Companion Encyclopaedia of the History and Philosophy of the Mathematical Sciences, London, 2003.

HEDRICK, H. L.: Theo van Doesburg. Propagandist and Practitioner of the Avant-Garde, 1909-1923, Ann Arbor, Michigan, 1980.

HILHORST, C.: "Bart van der Leck", en De Stijl: The Formative Years. 19171922, Cambridge, 1982.

__ "Bart van der Leck". En: http://www.snap-dragon.com/bart_van_der_leck.htm.

HOEK, E.: "Piet Mondriaan", en Beginjaren van De Stijl 1917-1922, Utrecht, 1982.

JAFFE, Hans L.C.: De Stijl, London, 1970.

JOHNSON, S. \& TODD, H. J. \& CHALMERS, A. \& WALKER, J.: English Dictionary, Boston, 1835.

JOHNSON, D. L. \& LANGMEAD, D.: Makers of 20th-Century Modern Architecture: A Bio-Critical Sourcebook, London, 1997. 
KRUGER, R.: "Art in the Fourth Dimension: Giving Form to Form - The Abstract Paintings of Piet Mondrian". Spaces of Utopia: An Electronic Journal, no. 5, 2007, pp. 23-35.

LEE, R.: Tangram, Málaga, 2009.

LLIBRE, J.: El Tangram de los ocho elementos, Barcelona, 1977.

MEGGS, P. B. \& PURVIS, A. W.: Meggs' History of Graphic Design, Hoboken, New Yersey, 2000.

MORRISON, G. S.: Educación infantil, Madrid, 2005.

OVERY, P.: De Stijl, London, 1991.

OXENAAR, R. W. D.: "Van der Leck y De Stijl. 1916-1920”, en De Stijl: 19171931. Visiones de utopía, Madrid, 1982.

PETERSEN, A.: Mondrian, van der Leck, van Doesburg. Obra sobre papel, Valencia, 1991.

VINCENT, R.: Geometry of the Golden Section, Marseille, 2007.

WARNCKE, C. P.: El arte de la forma ideal. De Stijl 1917-1931, Köln, 1993.

WHITE, M.: De Stijl and Dutch Modernism, Manchester, 2003.

YOUNG, A.: Dada and After: Extremist Modernism and English Literature, Manchester, 1983.

ZEVI, B.: Poética de la arquitectura neoplástica, Buenos Aires, 1960. 